\title{
Rational Conchoid and Offset Constructions: Algorithms and Implementation
}

\author{
Juana Sendra ${ }^{1,2}$, David Gómez ${ }^{2}$, and Valerio Morán ${ }^{3}$ \\ ${ }^{1}$ Dpto. de Matemática Aplicada a las TIC, Universidad Politécnica de Madrid, \\ Madrid, Spain \\ jsendra@etsist.upm . es \\ ${ }^{2}$ Research Center on Software Technologies and Multimedia Systems \\ for Sustainability, Madrid, Spain \\ david.gomezs@upm.es
}

${ }^{3}$ ETSI Sistemas de Telecomunicación, Universidad Politécnica de Madrid, Spain vmoran@alumnos.upm.es

\begin{abstract}
This paper is framed within the problem of analyzing the rationality of the components of two classical geometric constructions, namely the offset and the conchoid to an algebraic plane curve and, in the affirmative case, the actual computation of parametrizations. We recall some of the basic definitions and main properties on offsets (see [13]), and conchoids (see [15]) as well as the algorithms for parametrizing their rational components (see [1] and [16], respectively). Moreover, we implement the basic ideas creating two packages in the computer algebra system Maple to analyze the rationality of conchoids and offset curves, as well as the corresponding help pages. In addition, we present a brief atlas where the offset and conchoids of several algebraic plane curves are obtained, their rationality analyzed, and parametrizations are provided using the created packages.
\end{abstract}

\section{Introduction}

In this paper we deal with two different geometric constructions that appear in many practical applications, where the need of proving rational parametrizations as well as automatized algorithmic processes is important. On one side we consider offset varieties and on the other conchoid varieties. Offsets varieties have been extensively applied in the field of computer aided geometric design (see $[5],[3],[4])$, while conchoids varieties appears in several of practical applications, namely the design of the construction of buildings, in astronomy [6], in electromagnetism research [20], optics, physics, mechanical engineering and biological engineering [7], in fluid mechanics [19], etc.

The intuitive idea of these geometric constructions is the following. Let $\mathbb{C}$ be the field of complex numbers (in general, one can take any algebraically closed field of characteristic zero), and let $\mathcal{C}$ be an irreducible hypersurface in $\mathbb{C}^{n}$ (say 
$n=2$ or $n=3$, and hence $\mathcal{C}$ is a curve or a surface). Moreover, although it is not necessary for the development of the theory, in practice one considers that $\mathcal{C}$ is real (i.e. there exists at least one regular real point on $\mathcal{C}$ ). The offset variety to $\mathcal{C}$ at distance $d$ ( $d$ is a field element, in practice a non-zero real number), denoted by $\mathcal{O}_{d}(\mathcal{C})$, is the envelope of the system of hyperspheres centered at the points of $\mathcal{C}$ with fixed radius $d$ (see Fig.1, left); for a formal definition, see e.g. [1]. In particular, if $\mathcal{C}$ is unirational and $\mathcal{P}(\bar{t})$, with $\bar{t}=\left(t_{1}, \ldots, t_{n}\right)$, a rational parametrization of $\mathcal{C}$, the offset to $\mathcal{C}$ is the Zariski closure of the set in $\mathbb{C}^{n}$ generated by the expression $\mathcal{P}(\bar{t}) \pm d \frac{\mathcal{N}(\bar{t})}{\|\mathcal{N}(\bar{t})\|}$ where $\mathcal{N}(\bar{t})$ is the normal vector to $\mathcal{C}$ associated with $\mathcal{P}(\bar{t})$.

The conchoid construction is also rather intuitive. Given $\mathcal{C}$ as above (base variety) and a fixed point $A$ (focus), consider the line $\mathcal{L}$ joining $A$ (in practice the focus is real) to a point $P$ of $\mathcal{C}$. Now we take the points $Q$ of intersection of $\mathcal{L}$ with a hypersphere of radius $d$ centered at $P$. The Zariski closure of the geometric locus of $Q$ as $P$ moves along $\mathcal{C}$ is called the conchoid variety of $\mathcal{C}$ from focus $A$ at a distance $d$ and denoted by $\mathfrak{C}_{d}^{A}(\mathcal{C})$ (see Fig.1 right); for the geometric construction of the conchoid and, for a formal definition, see e.g. [15] and [10]. The Conchoid of Nicomedes and the Limaçon of Pascal are the two classic examples of conchoids, and the best known. They appear when the base curve is a line or a circle, respectively. Similarly, if $\mathcal{C}$ is unirational and $\mathcal{P}(\bar{t})$ is a rational parametrization of $\mathcal{C}$, then the conchoid is the Zariski closure of the set defined by the expression

$$
\mathcal{P}(\bar{t}) \pm d \frac{\mathcal{P}(\bar{t})-A}{\|\mathcal{P}(\bar{t})-A\|}
$$
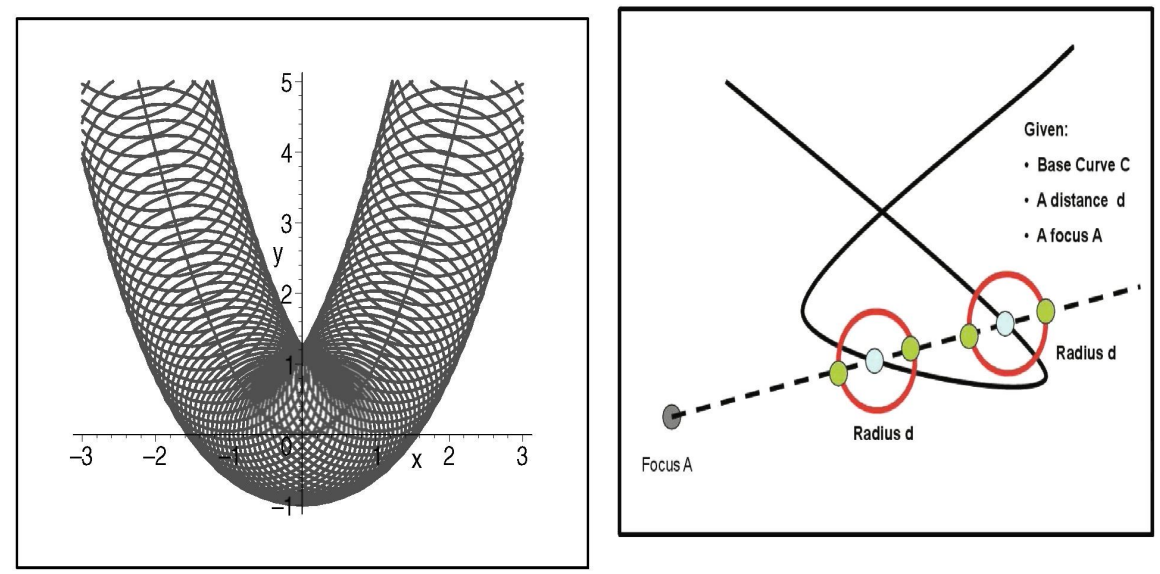

Fig. 1. Left: Construction of the offset to the parabola, Right: Geometric construction of the conchoid 
These two operations are algebro-geometric, in the sense that they create new algebraic sets from the given input objects. There is an interesting relation between the offset and the conchoid operations. Indeed, there exists a rational bijective quadratic map which transforms a given hypersurface $F$ and its offset $F_{d}$ to a hypersurface $G$ and its conchoidal $G_{d}$, and vice versa (see [11]).

The main difficulty when applying these constructions is that they generate much more complicated objects than the initial ones. There is a clear explosion of the degree of the hypersurface, singularity structure and the density of the defining polynomials (see e.g. [2], [13], [15]). As a consequence, in practice, the implicit equations are untractable from the computational point of view. This is one of the reasons why the use of parametric representations of offsets and conchoids are considered. Let us see an illustrating example. We consider the plane curve $\mathcal{C}$ defined by $y^{4}=x^{5}$. Its offset has degree 12 and the polynomial defining it has 65 nonzero terms, and its infinity norm is 300781250 . However, the offset can be parametrized by radicals (see [17], [18]) as $\left(t^{4}, t^{5}\right) \pm \frac{d}{\sqrt{25 t^{8}+16 t^{6}}}\left(-5 t^{4}, 4 t^{3}\right.$ ). On the other, the offset can be rationally parametrized as

$$
\begin{gathered}
\left(\frac{1}{625} \frac{\left(t^{2}-1\right)\left(16 t^{8}-32 t^{6}+625 d t^{4}+32 t^{2}-16\right)}{t^{4}\left(t^{2}+1\right)}\right. \\
\left.\frac{2}{3125} \frac{-16 t^{12}+64 t^{10}-80 t^{8}+3125 d t^{6}+80 t^{4}-64 t^{2}+16}{t^{5}\left(t^{2}+1\right)}\right) .
\end{gathered}
$$

The paper is structured as follows. In Section 1 we recall some of the basic definitions and main properties on offsets and conchoids of algebraic plane curves (see [13], [15]). We provide algorithms to analyze the rationality of the components of these new objects (see [1], [16]), and in the affirmative case, rational parametrizations are given. In Section 2 we present the creation of two packages in the computer algebra system Maple to analyze the rationality of offset and conchoids curves respectively, whose procedures are based on the above algorithms, as well as the corresponding help pages. Finally, in Section 3, we illustrate the performance of the package by presenting a brief atlas where the offset and conchoids of several algebraic plane curves are obtained, with their rationality analyzed. Furthermore, in case of genus zero, a rational parametrization is computed. We have not done an theoretical analysis of the complexity of the implemented algorithms but the practical performance of the implementation provides answers, in reasonable time for curves of degree less than 5 .

\section{Parametrization Algorithms: Curve Case}

In this section we summarize the results on the rationality of the offsets and conchoids of curves, presented in [1], [16] respectively, by deriving an algorithm for parametrizing them. The treatment of surfaces can be found at [1], [12], [14] (offsets) [8], [9], [10] (conchoids). 
The rationality of the components of the offsets is characterized by means of the existence of parametrizations of the curve whose normal vector has rational norm, and alternatively by means of the rationality of the components of an associated curve, that is usually simpler than the offset. As a consequence, one deduces that offsets to rational curves behave as follows: they are either reducible with two rational components (double rationality), or rational, or irreducible and not rational.

For this purpose, we first introduce two concepts: Rational Pythagorean Hodograph and curve of reparametrization. Let $\mathcal{P}(t)=\left(P_{1}(t), P_{2}(t)\right) \in \mathbb{C}(t)^{2}$ be a rational parametrization of $\mathcal{C}$. Then, $\mathcal{P}(t)$ is RPH (Rational Pythagorean Hodograph) if its normal vector $\mathcal{N}(t)=\left(N_{1}(t), N_{2}(t)\right)$ satisfies that $N_{1}(t)^{2}+N_{2}(t)^{2}=m(t)^{2}$, with $m(t) \in \mathbb{C}(t)$. For short we will express this fact writing $\|\mathcal{N}(t)\| \in \mathbb{C}(t)$. On the other hand, we define the reparametrizing curve of $\mathcal{O}_{d}(\mathcal{C})$ associated with $\mathcal{P}(t)$ as the curve generated by the primitive part with respect to $x_{2}$ of the numerator of $x_{2}^{2} P_{1}^{\prime}\left(x_{1}\right)-P_{1}^{\prime}\left(x_{1}\right)+2 x_{2} P_{2}^{\prime}\left(x_{1}\right)$, where $P_{i}^{\prime}$ denotes the derivative of $P_{i}$. In the following, we denote by $\mathcal{G}_{\mathcal{P}}(\mathcal{C})$ the reparametrizing curve of $\mathcal{O}_{d}(\mathcal{C})$ associated with $\mathcal{P}(t)$. Summarizing the results in [1], one can outline the following algorithm for offsets.

\section{Algorithm: offset parametrization}

- GIVEN: a proper rational parametrization $\mathcal{P}(t)$ of a plane curve $\mathcal{C}$ in $\mathbb{K}^{2}$ and $d \in \mathbb{C}$

- DECIDE: whether the components of $\mathcal{O}_{d}(\mathcal{C})$ are rational.

- Determine: (in the affirmative case) a rational parametrization of each component.

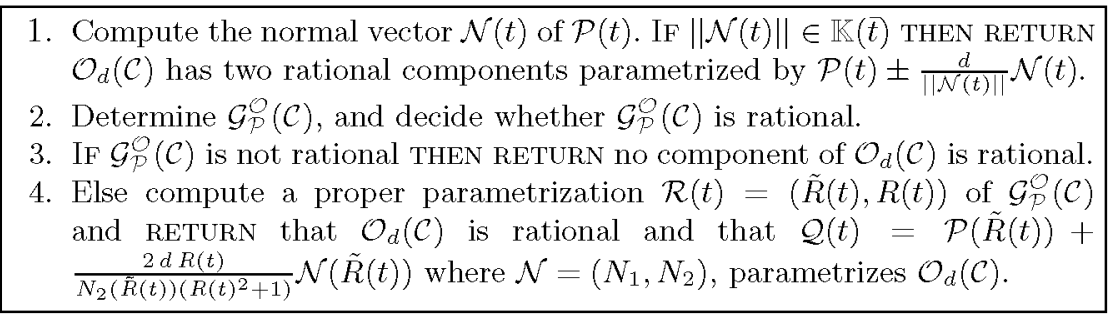

\section{The Conchoid Rationality Problem}

In [16], it is proved that conchoids having all their components rational can only be generated by rational curves. Moreover, it is shown that reducible conchoids to rational curves have always their two components rational (double rationality). From these results, one deduces that the rationality of the conchoid component, to a rational curve, does depend on the base curve and on the focus but not on the distance. To approach the problem we use similar ideas to those for offsets introducing the notion of reparametrization curve as well as the notion of rdf parametrization. The rdf concept allows us to detect the double rationality while the reparametrization curve is a much simpler curve than the conchoid, directly computed from the input rational curve and the focus, and that behaves 
equivalently as the conchoid in terms of rationality. As a consequence of these theoretical results [16] provides an algorithm to solve the problem. The algorithm analyzes the rationality of all the components of the conchoid and, in the affirmative case, parametrizes them. The problem of detecting the focuses from where the conchoid is rational or with two rational components is, in general, open.

We say that a rational parametrization $\mathcal{P}(t)=\left(P_{1}(t), P_{2}(t)\right) \in \mathbb{K}(t)^{2}$ of $\mathcal{C}$ is at rational distance to the focus $A=(a, b)$ if $\left(P_{1}(t)-a\right)^{2}+\left(P_{2}(t)-b\right)^{2}=$ $m(t)^{2}$, with $m(t) \in \mathbb{K}(t)$. For short, we express this fact saying that $\mathcal{P}(t)$ is rdf or $A$-rdf if we need to specify the focus. On the other hand, we define the reparametrization curve of the conchoid $\mathfrak{C}_{d}^{A}(\mathcal{C})$ associated to $\mathcal{P}(t)$, denoted by $\mathcal{G}_{\mathcal{P}}^{\mathfrak{C}}(\mathcal{C})$, as the primitive part with respect to $x_{2}$ of the numerator of $-2 x_{2}\left(P_{1}\left(x_{1}\right)-\right.$ a) $+\left(x_{2}^{2}-1\right)\left(P_{2}\left(x_{1}\right)-b\right)$.

\section{Algorithm: conchoid parametrization}

- GIVEN: a proper rational parametrization $\mathcal{P}(t)$ of a plane curve $\mathcal{C}$ in $\mathbb{K}^{2}$, a focus $A=(a, b)$, and $d \in \mathbb{C}$.

- DECIDE: whether the components of the conchoid $\mathfrak{C}_{d}^{A}(\mathcal{C})$ are rational.

- Determine: (in the affirmative case) a rational parametrization of each component.

1. Compute $\mathcal{G}_{p}^{\mathfrak{e}}(\mathcal{C})$.

2. If $\mathcal{G}_{\mathcal{P}}^{\mathfrak{e}}(\mathcal{C})$ is reducible RETURN that $\mathfrak{C}_{d}^{A}(\mathcal{C})$ is double rational and that $\mathcal{P}(t)+\frac{d}{ \pm\|\mathcal{P}(t)-A\|}(\mathcal{P}(t)-A)$ parametrize the two components.

3. Check whether the genus of $\mathcal{G}_{\mathcal{P}}^{\mathfrak{e}}$ is zero. If not, RETURN that $\mathfrak{C}_{d}^{A}(\mathcal{C})$ is not rational.

4. Compute a proper parametrization $\left(\phi_{1}(t), \phi_{2}(t)\right)$ of $\mathcal{G}_{p}^{\mathfrak{e}^{t}}$ and RETURN that $\mathfrak{C}_{d}^{A}(\mathcal{C})$ is rational and that $\mathcal{P}\left(\phi_{1}(t)\right)+\frac{d}{ \pm\left\|\mathcal{P}\left(\phi_{1}(t)\right)-A\right\|}\left(\mathcal{P}\left(\phi_{1}(t)\right)-A\right)$ parametrizes $\mathfrak{C}_{d}^{A}(\mathcal{C})$.

We can note that the rationality of the both constructions is not equivalent. For instance, if $\mathcal{C}$ is the parabola of equation $y_{2}=y_{1}^{2}$, that can be parametrized as $\left(t, t^{2}\right)$, the offset at distance $d$ is rational. However, the rationality of the conchoid of the parabola depends on the focus.

\section{Implementation of Conchoid and Offset Maple Packages and Help Pages}

In this section, we present the creation of two packages in the computer algebra system Maple, that we call Conchoid and Offset. These packages compute the implicit equation, and analyze the rationality and the reducibility of conchoids and offset curves respectively, providing rational parametrizations in case of genus zero. In addition, it allows us to display plots. These packages consist in several procedures that are based on the above parametrization algorithms. 
In the following, we give a brief description of the procedures and we show one of the help pages for one of the Maple functions. The procedure codes and packages are available in

http://www . euitt.upm.es/uploaded/docs_personales/sendra_pons_juana/ offsets_conchoids/Offset.zip

\subsection{Procedures of the Conchoid Package}

get ImplConch This procedure determines the implicit equation of the conchoid of an algebraic plane curve, given implicitely, at a fixed focus and a fixed distance. For this purpose, we use Gröbner basis to solve the system of equations consisting on the circle centered at generic point of the initial curve $\mathcal{C}$ and radius $d$, the straight line from the focus $A$ to the generic point of the initial curve $\mathcal{C}$, and the initial curve $\mathcal{C}$.

getParamConch Firstly this procedure checks whether the conchoid of a rational curve is irreducible or it has two rational components. For this purpose, we analyze whether a proper rational parametrization of the initial curve is RDF. In affirmative case, the procedure outputs a message indicating reducibility (the conchoid has two rational components) and a rational parametrization for each component is displayed. Otherwise, the conchoid is irreducible and the reparametrization curve is computed in order to study its rationality. In the affirmative case, it provides a rational parametrization by means of a rational parametrization of the reparametrizing curve and it outputs a message indicating irreducibility and rationality.

plotImplConch This procedure computes the conchoids curve using getImplConch procedure, and then it plots both the initial curve and its conchoid within the coordinates axes interval $[-a, a] \times[-a, a]$.

\subsection{Procedures of the Offset Package}

ImplicitOFF This procedure determines the implicit equation of the offset of a rational algebraic plane curve, given parametrically, at a fixed distance. For this purpose, since the algebraic system has three variables and one parameter (namely the distance), instead of Gröbner basis we simplify the computation by using resultants to solve the system of equations consisting on the circle centered at a generic point of the initial curve $\mathcal{C}$ and radius $d$, and the normal line at each point of $\mathcal{C}$.

OFFparametric This procedure analyzes the rationality of the offset of a rational plane curve. For this purpose, first it decides whether the offset is irreducible or it has two rational components. In case of reducibility, the procedure outputs 
a rational parametrization for each component, using the RPH concept. Otherwise, it checks whether the offset is rational or not. In the affirmative case, it provides a rational parametrization by means of a rational parametrization of the reparametrizing curve.

OFFplot This procedure computes the offset curve at a generic distance, $d$, and then replaces $d$ with fixed value, dist. Finally, it plots both the initial curve and its offset at a distance dist within the coordinates axes interval $[-a, a] \times[-a, a]$.

Once we have implemented the Offset/Conchoid procedure in Maple, we have created two packages containing them, called Conchoid and Offset, respectively.

Theoretically, to compute the implicit equation of either the conchoid or the offset, we use the incidence varieties introduced in [1] and [15], respectively. In the definition of this incidence variety an equation, to exclude extraneous factors, is introduced such that the Zariski closure of the projection is exactly the offset/conchoid curve. Therefore, by the theorem of the closure, Gröbner basis computation, and resultant when possible, provides the correct equation. In addition, one has to take into account that we are dealing with generic conchoids and generic offsets and therefore the specialization of the Gröbner basis or the resultant may fail. Nevertheless, since we have only one parameter there are only finitely many specializations; In particular, $d=0$ generates a bad specialization. Since $d=0$ is not interesting from the geometric construction point of view we are excluding this case. In addition, we have created the help pages associated to the procedures.

\section{Atlas of Conchoid and Offset Curves}

In this section we illustrate the previous results applying the packages Offset and Conchoid. We analyze the rationality of the offset and the conchoid of several classical rational curves, and in the case of rationality we compute rational parametrizations. We give a table summarizing the main details of the process for each geometric construction, such as the degree of the implicit equation, rational character and rational parametrization in case of genus zero. In case of Conchoids, the rationality depends on the focus, therefore in the table we study the rationality for different focus position, distinguishing if the focus is on the base curve or not. We don't include the implicit equation of the reparametrizing curve because of space limitations. The implicit equations, plots and more details of the computation of these atlas are available by contacting with the corresponding author. 
Table 1. Offsets Curves

\begin{tabular}{lcc}
\hline Base Curve & $\begin{array}{l}\text { Offset } \\
\text { Degree }\end{array}$ & Rationality \& Parametrization \\
\hline $\begin{array}{l}\text { Circle } \\
x_{2}^{2}+x_{1}^{2}-4\end{array}$ & 4 & $\begin{array}{c}\text { Double Rational } \\
\left( \pm \frac{(d \pm r) 2 t}{t^{2}+1}, \mp \frac{(d \pm r)\left(t^{2}-1\right)}{t^{2}+1}\right)\end{array}$ \\
\hline $\begin{array}{l}\text { Rarabolional } \\
x_{2}-x_{1}^{2}\end{array}$ & 6 & $\left(\frac{\left(t^{2}-1\right)\left(-t^{2}-1+4 d a t\right)}{4 a t\left(t^{2}+1\right)}, \frac{t^{6}-t^{4}-t^{2}+1+32 d t^{3} a}{16 a t^{2}\left(t^{2}+1\right)}\right)$
\end{tabular}

\section{Hyperbola}

$\frac{x_{1}^{2}}{16}-\frac{x_{2}^{2}}{9}-1$

8

Irreducible and non rational

$\begin{array}{lll}\text { Ellipse } & 8 & \text { Irreducible and non rational } \\ \frac{x_{1}^{2}}{25}+\frac{x_{2}^{2}}{16}-1 & & \end{array}$

\begin{tabular}{lcc}
\hline Cardioid & & Rational \\
$\left(x_{1}^{2}+4 x_{2}+\right.$ & 14 & $\left(\frac{\left(-9+t^{2}\right)\left(d t^{6}-117 d t^{4}+3456 t^{3}-1053 d t^{2}+729 d\right)}{\left(243 t^{2}+27 t^{4}+t^{6}+729\right)\left(t^{2}+9\right)}\right.$, \\
$\left.x_{2}^{2}\right)^{2}-16\left(x_{1}^{2}+\right.$ & & $\left.\frac{-18\left(d t^{6}-16 t^{5}-21 d t^{4}+864 t^{3}-189 d t^{2}-1296 t+729 d\right) t}{\left(243 t^{2}+27 t^{4}+t^{6}+729\right)\left(t^{2}+9\right)}\right)$ \\
$\left.x_{2}^{2}\right)$ & &
\end{tabular}

$\begin{array}{lll}\text { Three-leaved Rose } & \text { Irreducible and non rational } \\ \left(x_{1}^{2}+x_{2}^{2}\right)^{2}+x_{1}\left(3 x_{2}^{2}-x_{1}^{2}\right) & 14 & \end{array}$

\section{Trisectrix of Maclau-}

rin

Irreducible and non rational

$x_{1}\left(x_{1}^{2}+x_{2}^{2}\right)-\left(x_{2}^{2}-3 x_{1}^{2}\right)$

\section{Folium of Descartes}

$x_{1}^{3}+x_{2}^{3}-3 x_{1} x_{2}$

Irreducible and non rational

\section{Tacnode}

$2 x_{1}^{4}-3 x_{1}^{2} x_{2}+x_{2}^{2}-2 x_{2}^{3}+x_{2}^{4}$

Irreducible and non rational

\section{Epitrochoid}

$x_{2}^{4}+2 x_{1}^{2} x_{2}^{2}-34 x_{2}^{2}+x_{1}^{4}-\quad 10$

Irreducible and non rational

$34 x_{1}^{2}+96 x_{1}-63$

\section{Ramphoid Cusp}

$\begin{array}{ll}x_{1}^{4}+x_{1}^{2} x_{2}^{2}-2 x_{1}^{2} x_{2}-x_{1} x_{2}^{2}+ & 20 \quad \text { Irreducible and non rational } \\ x_{2}^{2} & \end{array}$

\begin{tabular}{lll} 
Lemniscata $\quad$ of & & \\
Bernoulli & 16 & Irreducible and non rational \\
$\left(x_{1}^{2}+x_{2}^{2}\right)^{2}-4\left(x_{1}^{2}-x_{2}^{2}\right)$ & & \\
\hline
\end{tabular}


Table 2. Conchoids Curves

\begin{tabular}{|c|c|c|}
\hline Curve $\mathcal{C}$ & $\begin{array}{l}\text { Focus Curve } \mathrm{F} \\
\text { Focus Conch. A }\end{array}$ & $\begin{array}{l}\text { Conchoid } \\
\text { Parametrization }\end{array}$ \\
\hline Circle & $\begin{array}{l}\mathrm{A}=\mathrm{F}=(0,0) \\
\mathrm{A}=(-2,0) \in \mathcal{C} \\
\mathrm{A}=(-4,0) \notin \mathcal{C}\end{array}$ & $\begin{array}{c}\left(\frac{-2\left(-1+t^{2}\right) \pm\left(1-t^{2}\right)}{1+t^{2}}, \frac{4 t \pm 2 t}{1+t^{2}}\right) \\
\left(\frac{3 t^{4}-12 t^{2}+1}{1+2 t^{2}+t^{4}}, \frac{2 t\left(-3+5 t^{2}\right)}{1+2 t^{2}+t^{4}}\right) \mathbf{R} \\
\text { NR }\end{array}$ \\
\hline Parabola & $\begin{array}{c}\mathrm{A}=\mathrm{F}=(0,1 / 4) \\
\mathrm{A} \in \mathcal{C}, \mathrm{A}=(0,0) \\
\mathrm{A}=(0,-2) \notin \mathcal{C}\end{array}$ & $\begin{array}{c}\left(t \pm \frac{4 t}{1+4 t^{2}}, t^{2} \pm \frac{4 t^{2}-1}{1+4 t^{2}}\right) \mathbf{D R} \\
\left(\frac{2 t+2 t^{3}+1-2 t^{2}+t^{4}}{\left(-1+t^{2}\right)\left(1+t^{2}\right)}, \frac{2 t\left(2 t+2 t^{3}+1-2 t^{2}+t^{4}\right)}{(-1+t)^{2}(1+t)^{2}\left(1+t^{2}\right)}\right) \mathbf{R} \\
\mathbf{N R}\end{array}$ \\
\hline Hyperbola & $\begin{array}{l}\mathrm{A}=\mathrm{F}=(5,0) \\
\mathrm{A}=(-4,0) \in \mathcal{C} \\
\mathrm{A}=(0,0) \notin \mathcal{C}\end{array}$ & $\begin{array}{c}\left(\frac{-2\left(9+t^{2}\right)}{3 t} \pm \frac{2(-t-6)(2 t+3)}{45+24 t+5 t^{2}}\right. \\
\left.\frac{t^{2}-9}{2 t} \pm \frac{3\left(t^{2}-9\right)}{45+24 t+5 t^{2}}\right) \mathbf{D R} \\
\left(\frac{\left(45 t^{6}+129 t^{4}+311 t^{2}+27\right)}{\left(1+t^{2}\right)\left(-9+t^{2}\right)\left(9 t^{2}-1\right)}, \frac{2\left(-63+81 t^{4}-82 t^{2}\right) t}{\left(1+t^{2}\right)\left(-9+t^{2}\right)\left(9 t^{2}-1\right)}\right) \mathbf{R} \\
\mathbf{N R}\end{array}$ \\
\hline Ellipse & $\begin{array}{l}\mathrm{A}=\mathrm{F}=(3,0) \\
\mathrm{A}=(0,4) \in \mathcal{C} \\
\mathrm{A}=(0,0) \notin \mathcal{C}\end{array}$ & $\begin{array}{c}\left(\frac{5\left(t^{2}-1\right)}{t^{2}+1} \pm \frac{t^{2}-4}{t^{2}+4}, \frac{8 t}{t^{2}+1} \pm \frac{4 t}{t^{2}+4}\right) \mathbf{D R} \\
\left(\frac{\left(1-t^{2}\right)\left(100 t+100 t^{3}+4 t^{4}+17 t^{2}+4\right)}{\left(4 t^{4}+17 t^{2}+4\right)\left(1+t^{2}\right)},\right. \\
\left.\frac{2\left(-58 t^{4}+8 t^{6}-58 t^{2}+8-4 t^{5}-17 t^{3}-4 t\right)}{\left(4 t^{4}+17 t^{2}+4\right)\left(1+t^{2}\right)}\right) \mathbf{R} \\
\mathbf{N R}\end{array}$ \\
\hline Cardioid & $\begin{array}{l}\mathrm{A}=(0,0) \in \mathcal{C} \\
\mathrm{A}=(-9,0) \notin \mathcal{C}\end{array}$ & $\begin{array}{c}\left(\frac{-1024 t^{3}}{\left(16 t^{2}+1\right)^{2}} \pm \frac{-8 t}{16 t^{2}+1}\right. \\
\left.\frac{-128 t^{2}\left(16 t^{2}-1\right)}{\left(16 t^{2}+1\right)^{2}} \pm \frac{1-16 t^{2}}{16 t^{2}+1}\right) \mathbf{D R} \\
\mathbf{N R}\end{array}$ \\
\hline $\begin{array}{l}\text { Three- } \\
\text { leaved Rose }\end{array}$ & $\begin{array}{l}\mathrm{A}=(0,0) \in \mathcal{C} \\
\mathrm{A}=(-2,0) \notin \mathcal{C}\end{array}$ & $\begin{array}{c}\left(\frac{2\left(t^{4}-6 t^{2}+9\right) t^{2}(t-1)(t+1)}{\left(t^{4}+2 t^{2}+1\right)^{2}}, \frac{4 t^{3}\left(t^{4}-6 t^{2}+9\right)}{\left(t^{4}+2 t^{2}+1\right)^{2}}\right) \mathbf{R} \\
\mathbf{N R}\end{array}$ \\
\hline $\begin{array}{l}\text { Trisectrix of } \\
\text { Maclaurin }\end{array}$ & $\begin{array}{l}\mathrm{A}=(0,0) \in \mathcal{C} \\
\mathrm{A}=(-4,0) \notin \mathcal{C}\end{array}$ & $\begin{array}{c}\left(\frac{-2\left(-5 t^{2}+2 t^{4}+1\right)}{\left(t^{4}+2 t^{2}+1\right)}, \frac{-4 t\left(-5 t^{2}+2 t^{4}+1\right)}{\left(t^{4}+2 t^{2}+1\right)\left(t^{2}-1\right)}\right) \mathbf{R} \\
\mathbf{N R}\end{array}$ \\
\hline $\begin{array}{l}\text { Folium of } \\
\text { Descartes }\end{array}$ & $\begin{array}{l}\mathrm{A}=(0,0) \in \mathcal{C} \\
\mathrm{A}=(-1,-1) \notin \mathcal{C}\end{array}$ & $\begin{array}{c}\left(\frac{\left(-6 t+6 t^{5}+t^{6}-3 t^{4}+3 t^{2}-1+8 t^{3}\right)(t-1)(t+1)}{\left(t^{2}+1\right)\left(t^{6}-3 t^{4}+3 t^{2}-1+8 t^{3}\right)},\right. \\
\left.\frac{2\left(-6 t+6 t^{5}+t^{6}-3 t^{4}+3 t^{2}-1+8 t^{3}\right) t}{\left(t^{2}+1\right)\left(t^{6}-3 t^{4}+3 t^{2}-1+8 t^{3}\right)}\right) \mathbf{R} \\
\mathbf{N R}\end{array}$ \\
\hline Tacnode & $\begin{array}{l}\mathrm{A}=(0,0) \in \mathcal{C} \\
\mathrm{A}=(0,1) \in \mathcal{C}\end{array}$ & $\begin{array}{l}\text { NR } \\
\text { NR }\end{array}$ \\
\hline Epitrochoid & $\begin{array}{l}\mathrm{A}=(3,0) \in \mathcal{C} \\
\mathrm{A}=(0,0) \notin \mathcal{C}\end{array}$ & $\begin{array}{c}\left(\frac{-7 t^{4}+288 t^{2}+256}{\left(t^{2}+16\right)^{2}} \pm \frac{16-t^{2}}{t^{2}+16}\right. \\
\left.\frac{-16 t\left(5 t^{2}-16\right)}{\left(t^{2}+16\right)^{2}} \pm \frac{(-8 t)}{t^{2}+16}\right) \mathbf{D R} \\
\mathbf{N R}\end{array}$ \\
\hline $\begin{array}{l}\text { Ramphoid } \\
\text { Cusp }\end{array}$ & $\begin{array}{c}\mathrm{A}=(0,0) \in \mathcal{C} \\
\mathrm{A}=(-1,-1) \notin \mathcal{C}\end{array}$ & $\begin{array}{l}\text { NR } \\
\text { NR }\end{array}$ \\
\hline $\begin{array}{l}\text { Lemniscata } \\
\text { of Bernoulli }\end{array}$ & $\begin{array}{l}\mathrm{A}=(-1,-1) \in \mathcal{C} \\
\mathrm{A}=(-2,0) \in \mathcal{C}\end{array}$ & $\begin{array}{l}\text { NR } \\
\text { NR }\end{array}$ \\
\hline
\end{tabular}

DR Double Rational, R Rational, NR Irreducible and Non Rational 

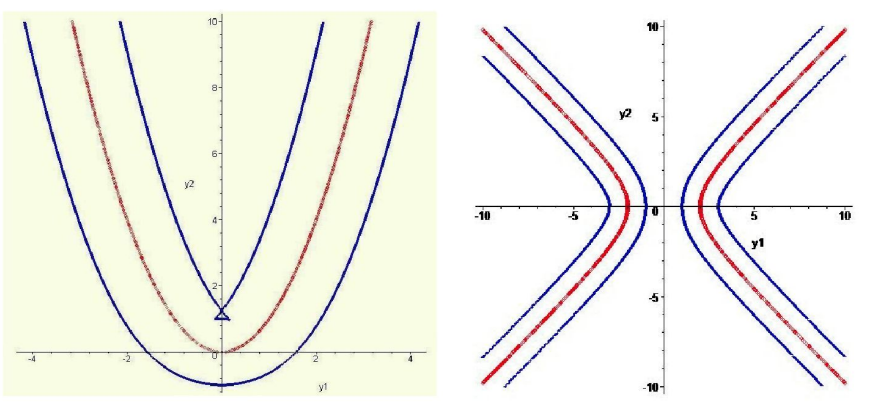

Fig. 2. Left: Parabola and the offset at $d=2$. Right: Hyperbola and the offset at $d=1.5$.
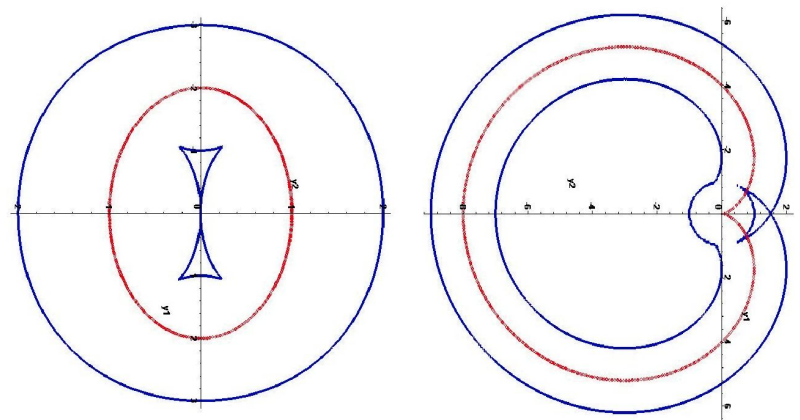

Fig. 3. Left: Ellipse and the offset at $d=1$. Right: Cardioid and the offset at $d=1$.
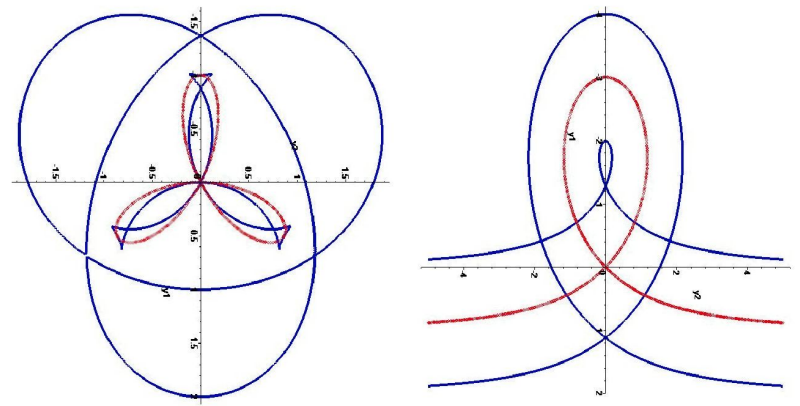

Fig. 4. Left: Three-leaved Rose and the offset at $d=1$. Right: Trisectrix of Maclaurin and the offset at $d=1$. 

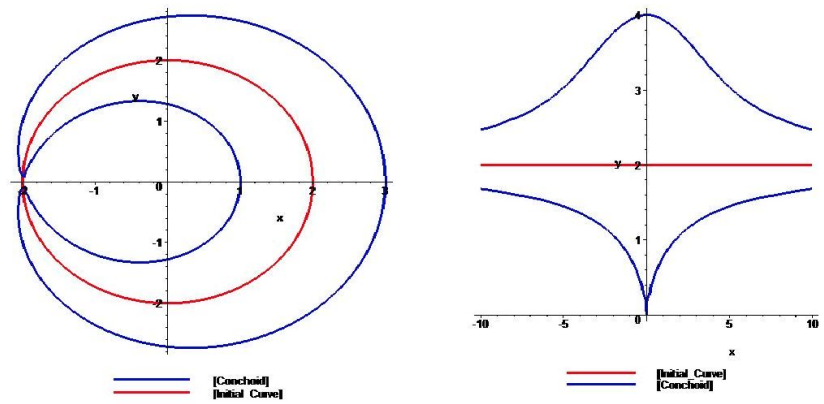

Fig. 5. Left: Circle and the conchoid at $A=(-2,0)$ and $d=1$ (Limaçon of Pascal). Right: Straight line and the conchoid at $A=(0,0)$ and $d=2$ (Conchoid of Nicomedes).
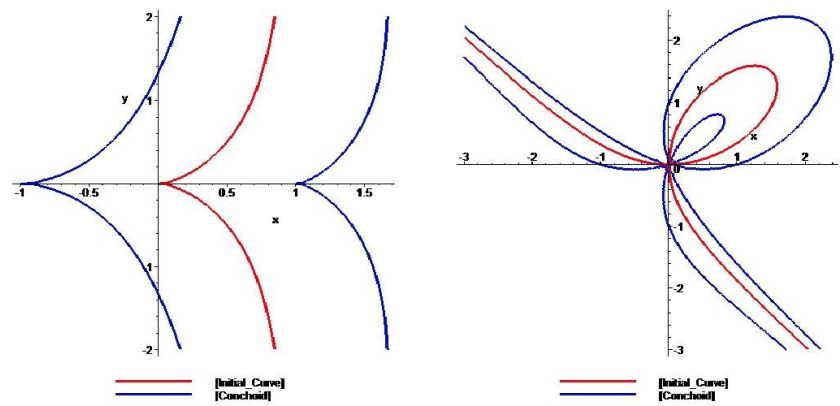

Fig. 6. Left: Conchoid of Sluze and the conchoid at $A=(-2,0)$ and $d=1$. Right: Folium of Descartes and the conchoid at $A=(0,0)$ and $d=2$.
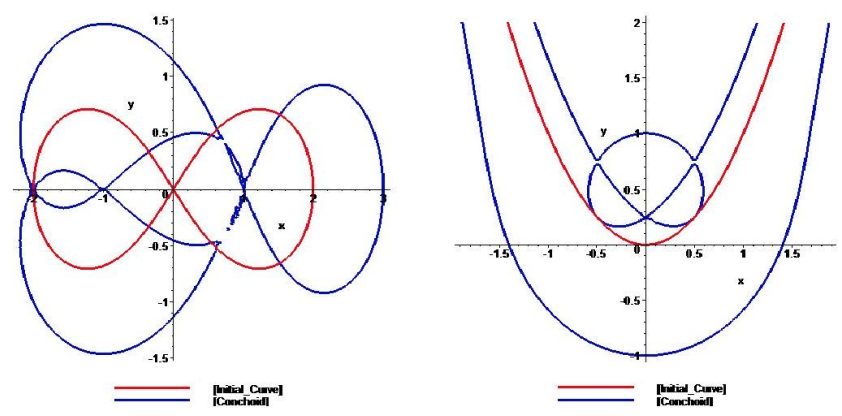

Fig. 7. Left: Lemniscata of Bernoulli and the conchoid at $A=(-2,0)$ and $d=1$. Right: Parabola and the conchoid at $A=(0,1 / 4)$ and $d=1$. 
Acknowledgements. Corresponding Author supported by the Spanish Ministerio de Economia y Competitividad under the Project MTM2011-25816-C02-01.

\section{References}

1. Arrondo, E., Sendra, J., Sendra, J.R.: Parametric Generalized Offsets to Hypersurfaces. Journal of Symbolic Computation 23(2-3), 267-285 (1997)

2. Arrondo, E., Sendra, J., Sendra, J.R.: Genus Formula for Generalized Offset Curves. Journal of Pure and Applied Algebra 136(3), 199-209 (1999)

3. Farouki, R.T., Neff, C.A.: Analytic Properties of Plane Offset Curves. Comput. Aided Geom. Des. 7, 83-99 (1990)

4. Farouki, R.T., Neff, C.A.: Algebraic Properties of Plane Offset Curves. Comput. Aided Geom. Des. 7, 100-127 (1990)

5. Hoffmann, C.M.: Geometric and Solid Modeling. Morgan Kaufmann Publis. (1993)

6. Kerrick, A.H.: The limaçon of Pascal as a basis for computed and graphic methods of determining astronomic positions. J. of the Instit. of Navigation 6, 5 (1959)

7. Menschik, F.: The hip joint as a conchoid shape. J. of Biomechanics 30(9) (September 1997) 971-3 9302622

8. Peternell, M., Gruber, D.: Conchoid surfaces of quadrics. Journal of Symbolic Computation (2013), doi:10.1016/j.jsc, 07.003

9. Peternell, M., Gruber, D., Sendra, J.: Conchoid surfaces of rational ruled surfaces. Comp. Aided Geom. Design 28, 427-435 (2011)

10. Peternell, M., Gruber, D., Sendra, J.: Conchoid surfaces of spheres. Comp. Aided Geom. Design 30(1), 35-44 (2013)

11. Peternell, M., Gotthart, L., Sendra, J., Sendra, J.: The Relation Between Offset and Conchoid Constructions. arXiv:1302.1859v2 [math.AG] (June 10, 2013)

12. Peternell, M., Pottmann, H.: A Laguerre geometric approach to rational offsets. Computer Aided Geometric Design 15, 223-249 (1998)

13. Sendra, J., Sendra, J.R.: Algebraic Analysis of Offsets to Hypersurfaces. Mathematische Zeitschrif 234, 697-719 (2000)

14. Sendra, J., Sendra, J.R.: Rationality Analysis and Direct Parametrization of Generalized Offsets to Quadrics. Applicable algebra in Engineering, Communication and Computing 11, 111-139 (2000)

15. Sendra, J., Sendra, J.R.: An Algebraic Analysis of Conchoids to Algebraic Curves. Applicable Algebra in Engineering, Communication and Computing 19, 413-428 (2008)

16. Sendra, J., Sendra, J.R.: Rational parametrization of conchoids to algebraic curves, Applicable Algebra in Engineering. Communication and Computing 21(4), 413-428 (2010)

17. Sendra, J.R., Sevilla, D.: Radical Parametrizations of Algebraic Curves by Adjoint Curves. Journal of Symbolic Computation 46, 1030-1038 (2011)

18. Sendra, J.R., Sevilla, D.: First Steps Towards Radical Parametrization of Algebraic Surfaces. Computer Aided Geometric Design 30(4), 374-388 (2013)

19. Sultan, A.: The Limaçon of Pascal: Mechanical Generating Fluid Processing. J. of Mechanical Engineering Science 219(8), 813-822 (2005) ISSN.0954-4062

20. Weigan, L., Yuang, E., Luk, K.M.: Conchoid of Nicomedes and Limaçon of Pascal as Electrode of Static Field and a Wavwguide of High Frecuency Wave. Progres. In: Electromagnetics Research Symposium, PIER, vol. 30, pp. 273-284 (2001) 